\title{
Choice responding following multiple schedule training
}

\author{
EUGENE L. EDMON, IRWIN LUCKI, and MICHAEL G. GRISHAM \\ University of Iowa, Iowa City, Iowa 52242
}

\begin{abstract}
Pigeons' choice responding on 10-sec interpolated probes was studied after baseline training on multiple variable-interval variable-interval schedules of food reinforcement. Unreinforced choice following training with three different relative reinforcement rates (Experiment 1), with a 3-ply multiple schedule (Experiment 2), and with three different relative reinforcement durations (Experiment 3) was examined. Least squares lines were fit to choice relative response rate and schedule relative response rate as functions of training relative reinforcement rate; choice slope was significantly greater than schedule slope in all three experiments. This result is counter to the prediction of Herrnstein's (1970) theory that these slopes should not differ. Luce's (1959) theory also failed to account for the data. It was concluded that choice responding was controlled by both approach to the stimulus associated with the smaller mean interreinforcer interval or the longer duration, and avoidance of the other stimulus.
\end{abstract}

Herrnstein's (1970) theory specifies the relation between response strength and reinforcement rate in the components of multiple and concurrent schedules. Since Herrnstein further proposes that "choice is nothing but behavior set into the context of other behavior" (p. 255), relative response frequency should be the same whether measured simultaneously or successively, once component response strengths are established by the schedule. That is, Herrnstein's equation for multiple-schedule relative response frequency is also the equation for choice relative response frequency following multiple-schedule training. This prediction, that schedule and choice relative rates should be equivalent functions of reinforcement, was evaluated by investigating choice responding on interpolated probes following asymptotic multiple-schedule training.

The choice probe results also permit further evaluation of the applicability of Luce's (1959) choice model in extinction tests. Luce (1977) has noted that matching on concurrent schedules is consistent with his model. However, Herrnstein and Loveland (1976), who employed a transfer test similar to that used here, found that choice results were more extreme than predicted by Luce's model. Herrnstein and Loveland (1976) used the ratios of concurrent-schedule relative response frequencies to define a scale of values for Luce's model, and then

The authors wish to thank Edward A. Wasserman and Gary Lucas for comments on the manuscript. Portions of the data were presented at the Annual Meeting of the Midwestern Psychological Association, Chicago, 1979. Michael G. Grisham is now at Bell Laboratories, Holmdel, New Jersey. Reprints may be obtained from Eugene L. Edmon, Department of Psychology, University of Iowa, Iowa City, Iowa 52242. employed this scale in predicting choice. The question of interest here is whether or not the model applies when choice is predicted by a scale derived from previous choice tests, not schedule responding. If the theory applies in this situation, we would have a basis for a ratio scale of relative response strength as a function of relative reinforcement rate for multipleschedule training, which is what the matching law provides for concurrent-schedule training. Nevin (1974) has reviewed arguments for the desirability of such a scale, with response strength conceived as a psychological construct that varies with parameters of reinforcement.

Experiment 1 studied choice following three different relative reinforcement rates. To extend the generality of the results and technique, Experiment 2 measured choice following 3-ply multiple-schedule training, and Experiment 3 studied probe responding following three different relative reinforcement durations.

\section{EXPERIMENT 1}

\section{Method}

Subjects. Four experimentally naive homing pigeons obtained from a local breeder were maintained at $80 \% \pm 10 \mathrm{~g}$ of their freefeeding weights. The subjects were given supplemental feeding of mixed grain after each daily session if necessary.

Apparatus. The two side keys, $16.5 \mathrm{~cm}$ apart center-to-center, were used in four identical three-key pigeon chambers. The chamber interior was $29 \mathrm{~cm}$ long $\times 38 \mathrm{~cm}$ wide $\times 37 \mathrm{~cm}$ high. The reinforcer was $3-\mathrm{sec}$ access to mixed grain presented through a $5 \times 5.5 \mathrm{~cm}$ opening equidistant from the two $2.5-\mathrm{cm}$ side keys and $13 \mathrm{~cm}$ below them on the response panel. The only chamber illumination during reinforcement was from an ESB24 lamp at $24 \mathrm{~V}$ in the BRS/LVE No. 114-10 grain hopper. Response switch closure required a force of .15 to $.20 \mathrm{~N}$. Industrial Electronics 
Engineers Series 10 readout projectors with No. 44 lamps at $5 \mathrm{~V}$, which presented red or green colors, were mounted behind the translucent Plexiglas response keys. The houselight was a No. 33 lamp at $5 \mathrm{~V}$ in a housing that directed the light upward. Ventilation and masking noise were provided by an exhaust fan. Experimental control and data collection were managed from a separate room by a PDP $-8 / F$ computer (Digital Equipment Corporation) using the SKED software system to control a solid-state interface.

Procedure. Keypeck responding was established by the method of successive approximations. Shaping was followed by 10 days of training to peck whichever side key was illuminated, with each peck reinforced and $\mathbf{4 2}$ reinforcers available each day. Red $(R)$ and green $(G)$ stimuli alternated with each response during this pretraining, and the right (R) or left (L) key was lit by the appropriate color according to the repeating sequence RLRRLRLL.

Pretraining was followed by exposure to the following sequence of schedules: multiple VI $15 \mathrm{sec}(R)$ VI $60 \mathrm{sec}(\mathrm{G})$; multiple VI $60 \mathrm{sec}(\mathrm{G})$ VI $120 \mathrm{sec}$ (R); multiple VI $15 \mathrm{sec}$ (G) VI $120 \mathrm{sec}$ (R); multiple VI $15 \mathrm{sec}(\mathrm{R})$ VI $60 \mathrm{sec}(\mathrm{G})$. The schedules were in effect until responding no longer changed systematically; this required $32,40,22$, and 34 sessions, respectively. Eight days of choice testing were then given. In parentheses above are the color assignments for Pigeons 242 and 243; Pigeons 245 and 251 had the reverse assignment. Red and green component stimuli strictly alternated and appeared on the keys in the same sequence used in pretraining. Thus, key position and color were uncorrelated and the discrimination could not be learned on the basis of position.

Constant conditional probability schedules were produced by randomly determining, every $3 \mathrm{sec}$, whether to make a reinforcer available, with the probabilities of .2 (VI $15 \mathrm{sec}$ ), .05 (VI $60 \mathrm{sec}$ ), or .025 (VI $120 \mathrm{sec}$ ). As in typical variable-interval schedules, a reinforcer made available was held until collected. Scheduled reinforcers were cancelled by component change or availability of the next reinforcer. Sessions terminated after at least 40 reinforcers were earned and an equal number of 1 -min components of each schedule had occurred. Components were separated by a 6-sec blackout.

During the last eight sessions of each multiple schedule, all component changes had an interpolated $10-\mathrm{sec}$ presentation of both discriminative stimuli, one on each side key. Stimulus position strictly alternated between left and right keys on these probe trials. Thus, if red were on the left for a particular probe, it would be on the right in the following probe. Note that red or green on the right during the probes would equally of ten follow red or green on the right during the immediately previous schedule component. Reinforcement never occurred on probe trials, which were preceded and followed by 6-sec blackouts. The average number of probe trials per session over all pigeons for the sequence of schedules was $17,45,19,17$; the number of probe trials varied with the number of components required to earn 40 reinforcers.

\section{Results}

Schedule relative response rate as a function of obtained relative reinforcement rate is presented in Figure 1; both rates are averaged over the 8 days of choice testing. Starting nearest the lower left corner, the points represent the following relative response and reinforcement rates: VI $60 \mathrm{sec} /(\mathrm{VI} 60 \mathrm{sec}+\mathrm{VI}$ $15 \mathrm{sec}) ;$ VI $60 \mathrm{sec} /(\mathrm{VI} 60 \mathrm{sec}+\mathrm{VI} 120 \mathrm{sec}) ;$ VI $15 \mathrm{sec} /$ (VI $15 \mathrm{sec}+$ VI $120 \mathrm{sec}$ ). Schedule relative response rates showed the expected tendency toward undermatching (Lander \& Irwin, 1968; Reynolds, 1963), a result consistent with the reverse-S-shaped curve expected from Herrnstein's (1970) multiple schedule equation. The average slope of the least squares lines fit to the data was .72 .

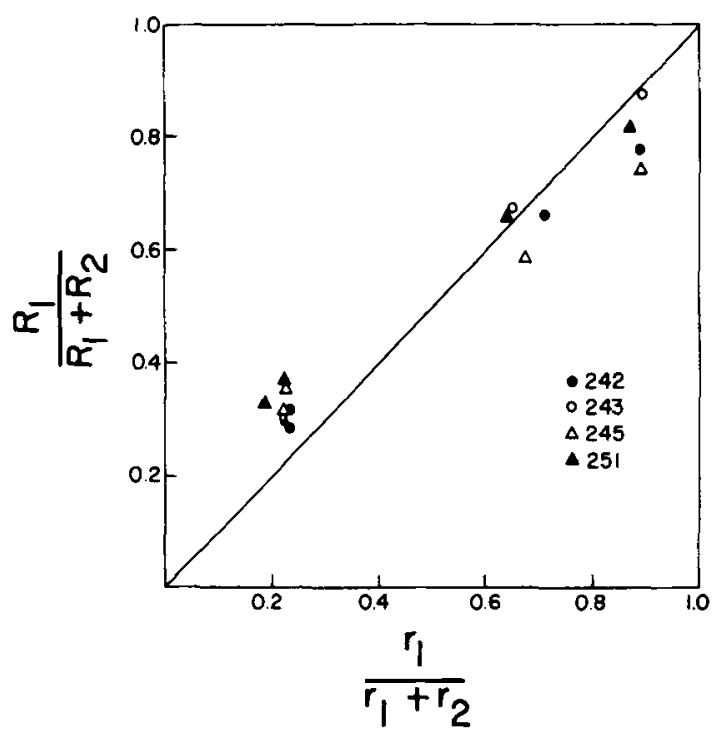

Figure 1. Schedule relative response rate as a function of relative reinforcement rate for the four 2 -ply multiple schedules employed in Experiment 1.

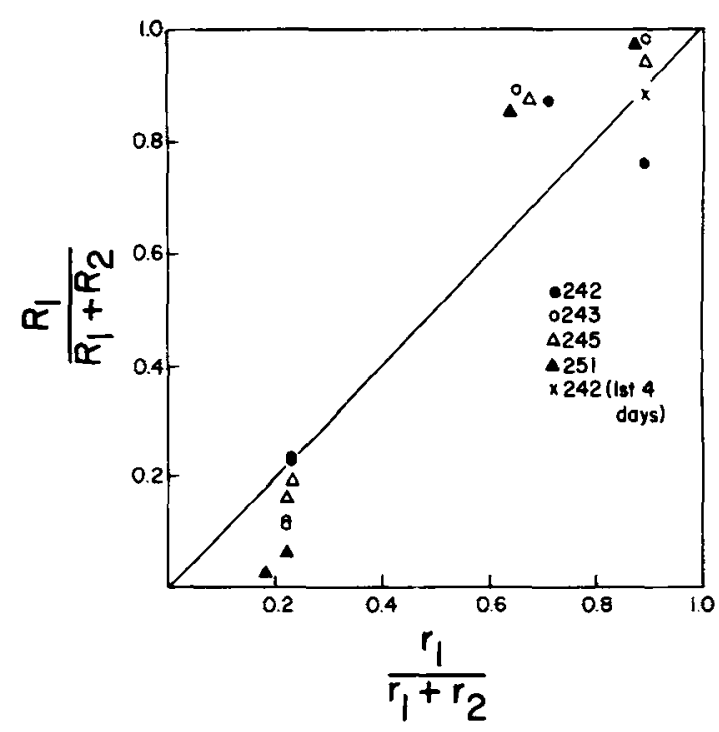

Figure 2. Choice relative response rate as a function of training relative reinforcement rate in Experiment 1.

Average choice probe relative response as a function of training relative reinforcement rate is presented in Figure 2. The order of the points corresponds to that in Figure 1. Choice relative rate tended to depart from the matching diagonal in the direction opposite to that found for schedule rate; the average least squares slope was 1.27 .

A $t$ test on the slope difference scores revealed a significant increase in choice slope over schedule slope $[\mathrm{t}(3)=4.58, \mathrm{p}<.05]$. It should be noted that although only three schedule relative reinforcement rates were investigated, the range of relative rates employed allows a valid comparison between schedule 
and choice slopes. It is not, however, being asserted that these data are necessarily best described by straight-line functions; the fits were done for comparison purposes.

In Figure 2 there are two points plotted for Pigeon 242 from the VI 15-sec VI 120-sec probe because this bird developed a strong position bias during the last 4 days of testing. Only the first 4 days' results were used in calculating the choice slope. For all birds, the second exposure to multiple VI $15 \mathrm{sec}$ VI $60 \mathrm{sec}$ replicated the schedule and choice relative rates of the initial exposure quite closely. Over all pigeons and reinforcement levels, 3,096 choice trials were given and only 36 had no response.

The choice data were examined to determine the applicability of Luce's (1959) choice model. If the assumptions of the model were appropriate for this procedure, choice relative rate from any one of the probes could be predicted by choice responding from the remaining two. Following Herrnstein and Loveland (1976), the ratios of relative response frequencies can be used to define a scale of value:

$$
V(A)=\frac{a}{a+b}
$$

"A" represents a reinforcement schedule and " $a$ " represents response rate on that schedule. With $A, B$, and $C$ referring to VI $15 \mathrm{sec}$, VI $60 \mathrm{sec}$, and VI $120 \mathrm{sec}$, respectively, the product rule of Luce's theory holds that:

$$
\frac{V(A)}{V(B)}=\frac{V(A)}{V(C)} \cdot \frac{V(C)}{V(B)}
$$

The predicted and obtained relative response frequencies for the VI 60 stimulus following multiple VI 15 -sec VI 60-sec training were: .50, .23 (242); .14, .11 (243); .32, .17 (245); .15, .04 (251). Obtained values are averages of the two determinations. These results are generally consistent with those of Herrnstein and Loveland (1976) in that obtained proportions are more extreme than those predicted.

\section{EXPERIMENT 2}

To study further the choice procedure and to provide a second test of Herrnstein's (1970) and Luce's (1959) theories, we investigated choice responding following 3-ply multiple schedule training in which the three different component schedules of Experiment 1 were combined. The 3-ply schedule was chosen because of our interest in the future use of the procedure for scaling the relation between reinforcement and generalized response strength (Peterson \& Premack, 1971) and because the 3-ply schedule would be much more efficient in terms of experimental time if it yielded results essentially equivalent to those of .Experiment 1. Thus, all of the results of Experiment 1 might be obtained following baseline training on only one schedule as opposed to three.

\section{Method}

Subjects. The same pigeons employed in Experiment 1 were used.

Apparatus. The apparatus was the same as in Experiment 1; only the two side keys were used.

Procedure. The pigeons were transferred to the 3-ply schedule immediately following the termination of Experiment 1. Blue (VI $15 \mathrm{sec}$ ), red (VI $60 \mathrm{sec}$ ), and green (VI $120 \mathrm{sec}$ ) stimuli were used. The schedule in effect and the associated side key were determined by sampling without replacement, so each schedule-position combination occurred equally often in each session. Position was thus irrelevant to the discrimination. Component duration was $1 \mathrm{~min}$ and sessions were terminated after 18 components; a 6-sec blackout occurred between components. After 34 training sessions, absolute response rates were not systematically changing and the probe procedure was put into effect for 8 sessions to measure choice on each of the three possible stimulus pairs. Probe trials followed each of the last 12 components of a session. For the first six probe trials, each of the six possible color-position combinations was randomly sampled without replacement; this sampling was then repeated for the next six probes.

\section{Results}

Figure 3 represents overall schedule relative response rate as a function of overall relative reinforcement rate, with relative rates averaged over the 8 choice days. The average slope of the least squares lines fit to these data was .48 . Figure 4 presents the pairwise choice relative response rates as a function of the training relative reinforcement rate for that pair of stimuli. The order of the points corresponds to those

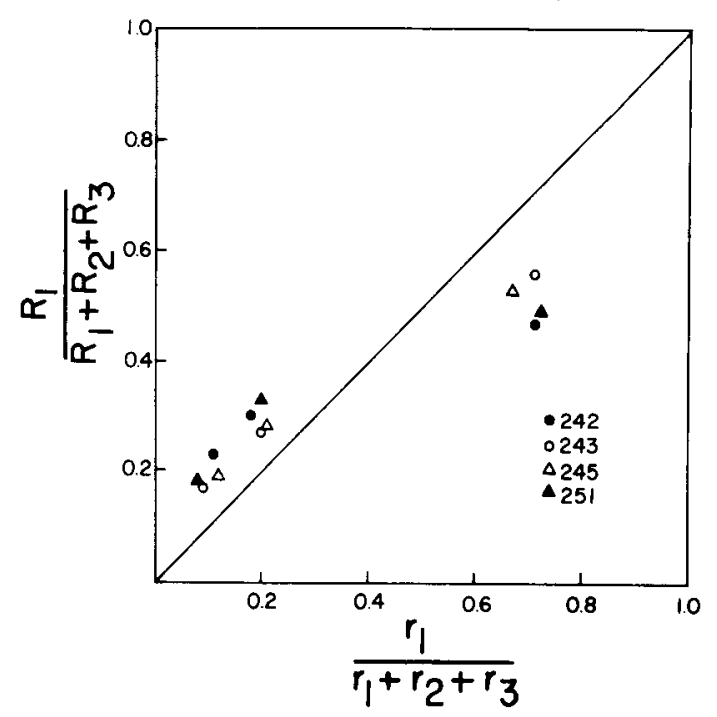

Figure 3. Overall schedule relative response rate as a function of overall relative reinforcement rate for the 3 -ply multiple schedule employed in Experiment 2. 


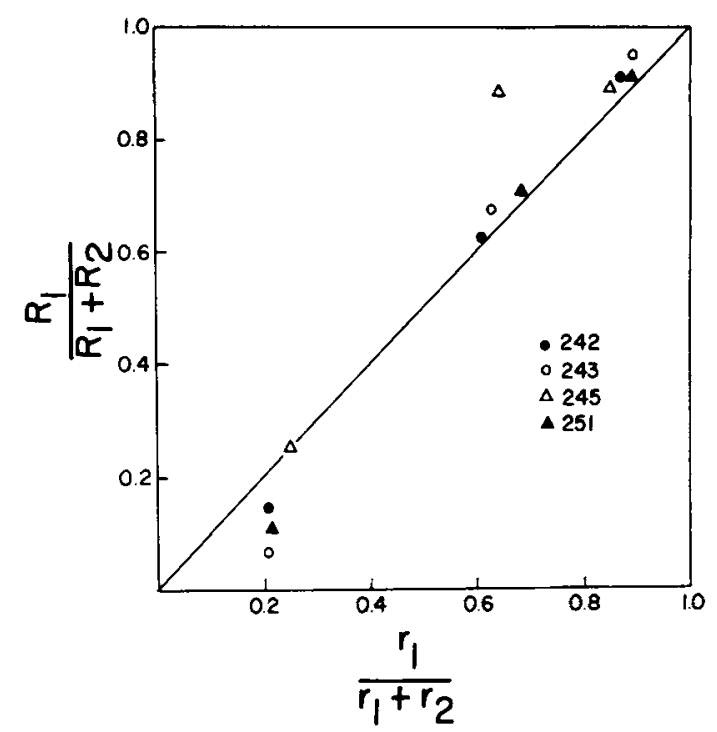

Figure 4. Pairwise relative response rate as a function of pairwise relative reinforcement rate with 3 -ply multiple schedule training.

in Figures 1 and 2. That is, nearest the lower left corner are points for the relative rates VI $60 \mathrm{sec} /(\mathrm{VI}$ $60 \mathrm{sec}+\mathrm{VI} 15 \mathrm{sec})$. Choice relative response rate was a monotonic function of relative reinforcement rate.

The average slope of lines fit to the choice data was 1.19. The obtained choice function was quite similar to that found in Experiment 1. The similarity is suggestive, implying that choice responding was not affected by the irrelevant alternative (Luce, 1959), but the small sample size here precluded an adequate statistical test. To compare schedule and choice slopes, lines were fit to pairwise schedule relative response and reinforcement rates, just as was done in Experiment 1; the average of these slopes was .53. The slope difference scores on the pairwise schedule and choice results revealed a significant increase in choice slope over schedule slope $[\mathrm{t}(3)=8.37, \mathrm{p}<.01]$.

The choice data were also examined for fit by Luce's (1959) model, following the method of prediction employed in Experiment 1. The predicted and obtained relative rates for VI $60 \mathrm{sec}$ in the VI $15-\mathrm{sec}$ VI 60 -sec choice were $.14, .14(242) ; .10, .07$ (243); $.48, .25(245) ; .19, .10(251)$. For three of the four pigeons, obtained values were more extreme than predicted, as might have been expected given the similarity of choice functions in the two experiments.

\section{EXPERIMENT 3}

It is also of interest to further establish the generality of the present procedure by investigating another parameter of reinforcement. Thus, Experiment 3 examined choice following multiple schedule training in which component reinforcement rates were equal but durations differed.

\section{Method}

Subjects. Four homing pigeons with previous schedule experience were maintained at $80 \% \pm 15 \mathrm{~g}$ of their free-feeding weights. The subjects were given supplemental feeding of mixed grain after each daily session if necessary.

Apparatus. The apparatus was the same as in Experiment 1.

Procedure. Three schedules were employed, all multiple VI $120 \mathrm{sec}$ VI $120 \mathrm{sec}$, with reinforcement duration values for the component schedules of $2 \mathrm{sec}(R)$ and $6 \mathrm{sec}(G), 3 \mathrm{sec}(R)$ and $2 \mathrm{sec}$ $(G)$, and $6 \sec (G)$ and $3 \sec (R)$. The letters in parentheses indicate the red or green colors associated with the components for Pigeons 266 and 271; Pigeons 269 and 272 had the opposite color assignment. The schedules were in effect for 16,43 , and 34 days, respectively. Scheduled reinforcers were cancelled by component change or availability of the next reinforcer. Sessions lasted for 481 -min components which were separated by 5 -sec blackouts. During training, the components strictly alternated and key position was determined by the repeating sequence RLRRLRLL; thus, position cues did not contribute to the discrimination.

During the last two sessions of each multiple schedule, each component change was preceded by a $10-\mathrm{sec}$ presentation of both discriminative stimuli, one on each key. Stimulus position strictly alternated between left and right keys on these choice probe trials. Thus, if red was on the left for a particular probe, it would be on the right in the following probe. Reinforcement never occurred on the probe trials, which were preceded and followed by 5 -sec blackouts.

\section{Results}

Schedule relative response rate as a function of relative reinforcement time is presented in Figure 5. Relative reinforcement time was calculated by dividing obtained total time on one schedule by obtained total time for both schedules and then averaging these values for the 2 choice days. From the lower left, the points are response and reinforcement values for $2 \mathrm{sec} /(2 \mathrm{sec}+6 \mathrm{sec}), 2 \mathrm{sec} /(2 \mathrm{sec}+3 \mathrm{sec})$, and $6 \mathrm{sec} /(3 \mathrm{sec}+6 \mathrm{sec})$. The average slope of lines fit to the schedule data was .36 .

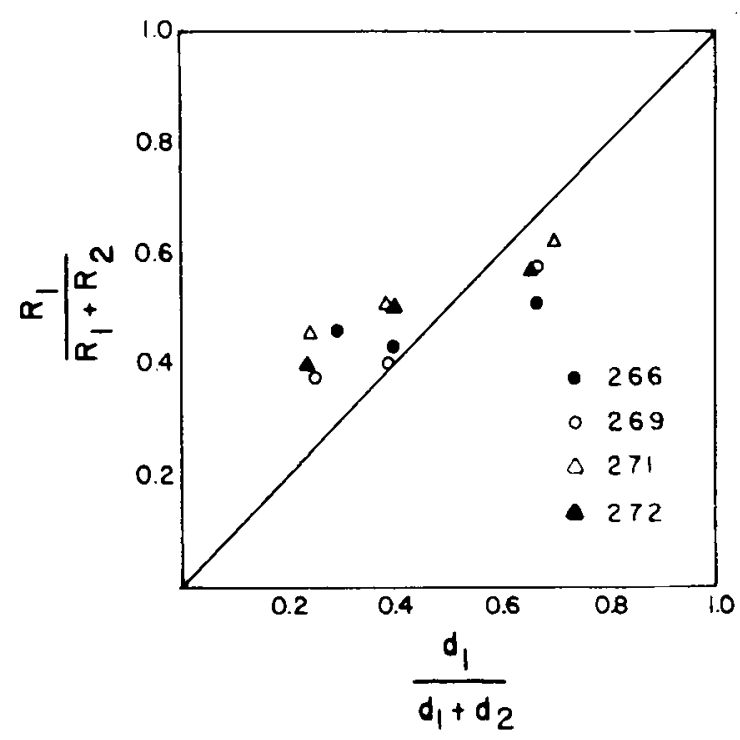

Figure 5. Schedule relative response rate as a function of relative reinforcement duration. 


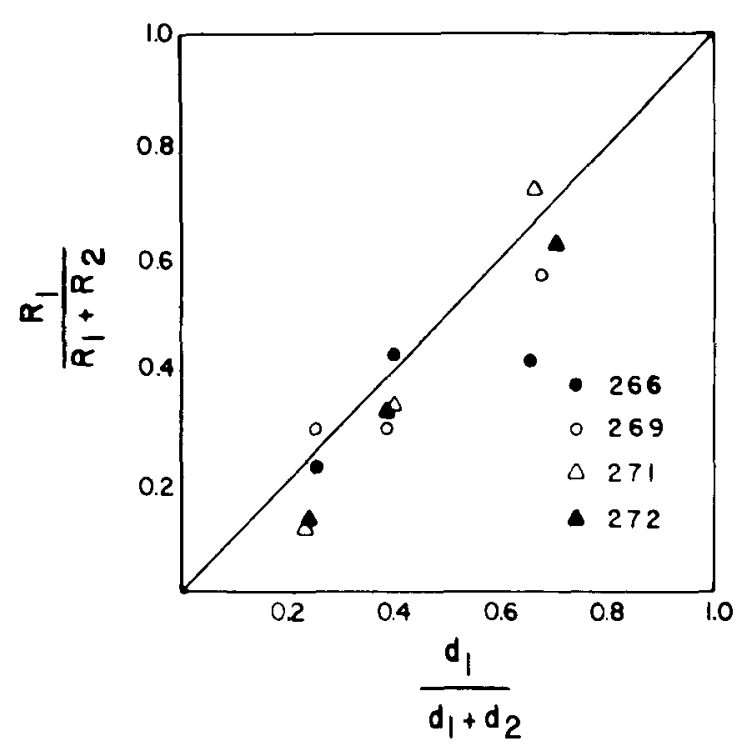

Figure 6. Choice relative response rate as a function of training relative reinforcement duration.

Relative response rates from the choice probes are presented in Figure 6, with points corresponding to those in Figure 5. Lines fit to these data have an average slope of .94 . The hypothesis that choice slope was less than or equal to schedule slope was rejected $[\mathrm{t}(3)=2.81, \mathrm{p}<.05]$.

Luce's model was again evaluated with these data. Relative rates from the $2-\mathrm{sec}$ vs. 6-sec and 3-sec vs. 6-sec choices were used to predict the remaining value. The predicted and obtained choice proportions were: $.174, .142(266) ; .246, .284(269) ; .198, .323$ (271); $.282, .335(272)$. For three of the four pigeons, predicted proportions were less than obtained proportions.

\section{GENERAL DISCUSSION}

The choice technique produced consistent and reproducible results. In each of the experiments, choice responding was a monotonic function of the reinforcement parameter employed. Also in each experiment, choice slopes were significantly greater than schedule slopes for best fitting lines.

Examination of the schedule results of all three experiments, as presented in Figures 1, 3, and 5, suggests that all might be adequately described by Herrnstein's (1970) multiple-schedule equation, which requires a reverse-S-shaped function symmetric about the main diagonal. However, the derivation from Herrnstein's theory that the choice and schedule functions should be the same is clearly not supported. This conclusion may be restricted to Experiments 1 and 2 , since Herrnstein did not explicitly extend his multiple schedule formulation to reinforcement duration. Either Herrnstein's multiple schedule equation or his choice assumption, or both, may be incorrect. Note that the choice assumption, as quoted earlier, plays an important role in the generality of Herrnstein's theory because it is that aspect of the theory which allows derivation of the prediction of matching on concurrent schedules from the single schedule equation.

Examination of the choice results of Experiments 1 and 2 might suggest that maximizing is the appropriate choice assumption to explain those functions. Note, however, that none of the results in Figure 2 or Figure 4 is what would be predicted by a maximizing model, which requires a step function from 0 to 1.0 for relative response rate at the relative reinforcement value of .5. Also, it certainly could not be concluded that the choice results of Experiment 3 represent maximizing. Again, the maximizing model fails to account for a single data point in the present set of experiments. Thus, given that an alternative choice assumption is not supported and that the multiple schedule equation may be in error, the present results do not permit certain rejection of Herrnstein's choice assumption.

Spealman and Gollub (1974) presented other evidence against the multiple-schedule equation in that the prediction of a direct relation between baseline reinforcement rate and magnitude of behavioral contrast effects was not supported. The equation also requires lower response rates on multiple schedule components of equal reinforcement density compared with the rate maintained by a single schedule of that density. McSweeney and Dericco (1976) and Spealman and Gollub (1974) have failed to support this prediction, finding essentially equal response rates in the two cases. The conclusion suggested is that Herrnstein's (1970) theory can apply only to simple and concurrent schedules, and the reason for this generality may be that, as Herrnstein noted, simple schedules actually are concurrent schedules in which the choice is between instrumental responding and other behavior. What is being suggested here is the restriction of the theory to situations in which behaviors are simultaneously available.

Luce (1977) noted that the matching often found on concurrent schedules is consistent with his choice theory (Luce, 1959). However, Herrnstein and Loveland (1976) found that the model did not do well in predicting choice responding on novel pairs of component stimuli from a complex concurrent training schedule. In that experiment, predictions for choice were derived from schedule responding.

In the present set of experiments, Luce's model did not predict well, even when choice predictions were derived from previous choice tests. Obtained choice proportions were generally more extreme than predictions for the reinforcement rate manipulation, 
with one exception. Obtained proportions were less extreme, with one exception, for the duration manipulation. The reason for this difference is not clear. An implication of the rejection of Luce's model is that the present procedure will not yield a ratio scaling between relative response strength and relative reinforcement. Unfortunately, from the present data we are not able to provide an adequate statistical evaluation of the fit of the model. Future work should provide such an evaluation.

A proposal made by Guttman (1959) would account for the fact that the choice slopes were greater than the schedule slopes in these experiments. Guttman found that peak shift occurred in the generalization gradient around a stimulus associated with a VI 60 -sec schedule after training on multiple VI $60 \mathrm{sec}$ VI $300 \mathrm{sec}$. He interpreted this result to mean that the stimulus associated with the VI $300-\mathrm{sec}$ schedule had become inhibitory in relation to the VI 60 -sec stimulus. Weisman (1969) reported inhibitory gradients around a multiple schedule stimulus associated with the longer mean interreinforcer interval, supporting Guttman's suggestion. In the present choice procedure, there may be tendencies to both approach the stimulus associated with the smaller mean interreinforcer interval or the longer duration and to avoid the other stimulus because of its inhibitory properties. This would account for the increase in choice slope over schedule slope. Note that Guttman's (1959) proposal of inhibitory effects comes not from an examination of his schedule results, but from the outcome of a transfer procedure, and the same is true for Weisman (1969) and ourselves. The choice procedure may provide a method for the future quantification of the proposed inhibitory effect.

\section{REFERENCES}

Guttman, N. Generalization gradients around stimuli associated with different reinforcement schedules. Journal of Experimental Psychology, 1959, 58, 335-340.

Herrnstein, R. J. On the law of effect. Journal of the Experimental Analysis of Behavior, 1970, 13, 243-266.

Herrnstein, R. J., \& Loveland, D. H. Matching in a network. Journal of the Experimental Analysis of Behavior, 1976, 26, 143-153.

LANDER, D. G., \& Irwin, R. J. Multiple schedules: Effects of the distribution of reinforcements between components on the distribution of responses between components. Journal of the Experimental A nalysis of Behavior, 1968, 11, 517-524.

LuCE, R. D. Individual choice behavior: A theoretical analysis. New York: Wiley, 1959.

Luce, R. D. The choice axiom after twenty years. Journal of Mathematical Psychology, 1977, 15, 215-233.

MCSWeEnex, F. K., \& Dericco, D. A. Rates of responding in the pigeon generated by simple and complex schedules which provide the same rates of reinforcement. Animal Learning \& Behavior, 1976, 4, 379-385.

Nevin, J. A. Response strength in multiple schedules. Journal of the Experimental Analysis of Behavior, 1974, 21, 389-408.

Peterson, J., \& Premack, D. A method for mapping stimulus distance into reinforcement value. Learning and Motivation, $1971,2,40-48$.

REYNOLDS, G. S. Some limitations on behavioral contrast and induction during successive discrimination. Journal of the Experimental A nalysis of Behavior, 1963, 6, 131-139.

SPE,AlMaN, R. D., \& GolluB, L. R. Behavioral interactions in multiple variable-interval schedules. Journal of the Experimental Analysis of Behavior, 1974, 22, 471-481.

Weisman, R. G. Some determinants of inhibitory stimulus control. Journal of the Experimental Analysis of Behavior, 1969 , $12,443-450$.

(Received for publication April 20, 1979; revision accepted October 22, 1979.) 